\title{
Phenomenological Model \\ of Hydrodynamic Cavitation Influence on Water Systems
}

\author{
Vladimir A. Kulagin*, \\ Tatiana A. Kulagina and Veronika V. Shelenkova \\ Siberian Federal University \\ 79 Svobodny, Krasnoyarsk, 660041, Russia
}

Received 11.09.2019, received in revised form 17.10.2019, accepted 01.11.2019

The rheological aspects of supercavitation flows in the flow part of technological equipment are considered. The description of the behavior of a cavitation spherical bubble in a liquid led to the formulation and solution of the boundary conjugation problem. It is shown that a cavitation spherical microbubble in its dynamics moves in a space structured by microturbulent vortices formed by the interference of rarefaction-compression waves resulting from the pulsation of cavitation microbubbles. As a result of the application of this model, the accuracy of calculating the supercavitation flow during the design of technological equipment for the treatment of heterogeneous media in the aquatic environment has increased.

Keywords: rheological aspects of supercavitation flows, boundary value problem, technological equipment, cavitation technology.

Citation: Kulagin V.A., Kulagina T.A., Shelenkova V.V. Phenomenological model of hydrodynamic cavitation influence on water systems, J. Sib. Fed. Univ. Eng. technol., 2019, 12(7), 818-829. DOI: 10.17516/1999-494X-0182.

(C) Siberian Federal University. All rights reserved

This work is licensed under a Creative Commons Attribution-NonCommercial 4.0 International License (CC BY-NC 4.0).

* Corresponding author E-mail address: v.a.kulagin@mail.ru 


\title{
Феноменологическая модель
}

\section{гидродинамического кавитационного воздействия на водные системы}

\author{
В.А. Кулагин, Т.А. Кулагина, В.В. Шеленкова \\ Сибирский федеральный университет \\ Россия, 660041, Красноярск, пр. Свободный, 79
}

\begin{abstract}
Рассмотрены реологические аспекты суперкавитационных потоков в проточной части технологического оборудования. Описание поведения кавитационного сферического пузырька в жидкости привело к формулировке и решению краевой задачи сопряжения. Показано, что кавитационный сферический микропузырек в своей динамике движется в пространстве, структурированном микротурбулентными вихрями, образованными интерференцией волн разрежения-сжатия, возникающих при пульсации кавитаџионных микропузырьков. В результате применения данной модели повысилась точность расчета суперкавитационного течения в прочессе проектирования технологического оборудования для обработки гетерогенных сред в водной среде.
\end{abstract}

Ключевые слова: реологические аспекты суперкавитационных потоков, краевая задача, технологическое оборудование, кавитационная технология.

\section{Введение}

Рассматриваемый феномен лежит в основе специфических свойств и связанных с ними явлений, происходящих в воде, подвергнутой механическому (гидродинамическому) воздействию. Модифицированная в результате гидромеханической обработки (или, как называют, активированная) вода способна интенсифицировать примерно на 30 \% ряд технологических процессов [1]. Здесь под термином «активированная вода» будем иметь в виду общепринятое понятие активной среды, т.е. вещества, в котором распределение частиц (атомов, молекул, ионов) по энергетическим состояниям не является равновесным и хотя бы для одной пары уровней энергии осуществляется инверсия населенности.

Активация твердых и жидких систем, приводящая к изменению их физических и химических свойств, реакционной способности, дефектной (примесной) структуры и т.п., может быть осуществлена различными внешними воздействиями: слабыми и сильными. К таким воздействиям можно, в частности, отнести механическую, магнитную, ультразвуковую обработку, радиационное воздействие (например, облучение гамма-квантами и ионными пучками), а также термообработку. Методы активации можно подразделить на методы, разрушающие образцы в целом (процесс диспергирования) и не разрушающие, а изменяющие только дефектную структуру.

Исследования гидромеханической обработки воды (как достаточно сильного воздействия) показали, что последующая активность воды проявляется как в макромасштабе, так и на микроуровнях (на молекулярном и субмолекулярном). Суть гидродинамического воздействия может быть сведена к действию двух механизмов: распространению ударных волн вблизи схлопывающегося кавитационного микропузырька и ударному действию кумулятивных микроструек при несимметричном коллапсе кавитационных микропузырьков [2-4]. Причем в

$$
-819-
$$


данном контексте способ получения кавитационных микропузырьков не имеет значения. Этим основным механизмам сопутствует повышение температуры и давления вблизи пузырька, делая локальную область около него уникальным реактором для проведения различных реакций и процессов. Предварительные результаты работы в этом направлении частично обобщены в монографиях [5-8]. В книге [5] рассмотрена краевая задача сопряжения для парогазового пузырька в жидкости, позволяющая путем детальных расчетов определить все особенности развития пузырька из зародыша и его схлопывания у твердой стенки. Там же на базе этой задачи рассмотрен частный случай для сферического пузырька в неподвижной жидкости. Результаты, полученные различными авторами, указывают на то, что при симметричном схлопывании пузырька создаются высокоинтенсивные поля давлений (до 5-10 тыс. атм.) и температур (до $\left.15000^{\circ} \mathrm{C}\right)[9-11]$.

Молекула $\mathrm{H}_{2} \mathrm{O}$ является самым легким и малым представителем химических и изоэлектронных гомологов. Ее свойства определяются максимально неоднородным распределением средней плотности электронов по молекуле, в результате чего на атомах молекулы $\mathrm{H}_{2} \mathrm{O}$ сосредоточены наибольшие эффективные заряды $[12,13]$. Уникальные свойства, парадоксы жидкой воды в большей степени объясняются наличием и развитостью водородных связей. В системе молекул $\mathrm{H}_{2} \mathrm{O}$ водородная связь носит кооперативный характер, во многом определяя структуру воды при различных внешних условиях. Водородные связи примерно в 10 раз сильнее межмолекулярных взаимодействий, характерных для большинства других жидкостей.

В общем случае можно сказать, что взаимодействия большого количества молекул, ансамблей молекул, организация той или иной структуры, определяющей свойства воды, и соответственно, ее реакционную способность, обуславливаются коллективными силами Ван-дерВаальса. Приближенно закон соответственных состояний может быть выражен уравнением Ван-дер-Ваальса в виде

$$
\frac{P V}{N k T}=\frac{V}{V-b}-\frac{a^{\prime} N}{V k T} .
$$

Силы Ван-дер-Ваальса известны как дисперсионные, дальнодействующие. Они захватывают области $>1000 \dot{\mathrm{A}}$ и определяют устойчивость той или иной структуры, физическую сорбцию и др.

Время релаксации для ряда процессов в воде при $T=20^{\circ} \mathrm{C} t=10^{-11}-10^{-13} \mathrm{c}$, а для некоторых - до $t \cong 10^{-14}$ с [9]. В связи с тем что продолжительность конечной стадии коллапса пузырька $t=10^{-9}-10^{-8}$ с, становятся возможными процессы передачи энергии и перезарядки с участием молекул воды, благородных и активных газов, а также диссоциация молекул воды.

Таким образом, под действием гидродинамической кавитации как сильного воздействия происходит разложение (механолиз) воды [1]. Возбужденная молекула воды, наряду с излучением и диссипацией избыточной энергии в тепло, может диссоциировать:

$$
\begin{aligned}
& \mathrm{H}_{2} \mathrm{O}^{*} \rightarrow \mathrm{H} \cdot+\dot{\mathrm{O}} \mathrm{H}^{*}, \\
& \dot{\mathrm{OH}}^{*} \rightarrow \dot{\mathrm{O}} \mathrm{H}+\mathrm{hv} .
\end{aligned}
$$


За счет механолиза воды на Н и ОН в результате кавитационного воздействия происходит увеличение концентрации $\mathrm{O}_{2}$ при протекании в процессе механохимических реакций типа

$$
\begin{aligned}
& \mathrm{OH}+\mathrm{OH} \rightarrow \mathrm{H}_{2} \mathrm{O}_{2}, \\
& \dot{\mathrm{OH}}+\mathrm{H}_{2} \mathrm{O}_{2} \rightarrow \mathrm{HO}_{2}+\mathrm{H}_{2} \mathrm{O}, \\
& \dot{\mathrm{OH}}+\mathrm{HO}_{2} \rightarrow \mathrm{H}_{2} \mathrm{O}+\mathrm{O}_{2} .
\end{aligned}
$$

Одновременно изменяется структуры воды с образованием свободных водородных связей, что обуславливает ее повышенную активность и реагентную способность.

В случае водных систем активация, кроме механолиза воды, заключается в изменении степени равномерности распределения примесей по объему системы, агрегации и дезагрегации (диспергирования) примесей, а также в изменении их активного состояния. Важнейшей особенностью водных систем, в частности, является гетерогенность по примесям, которая в процессе кавитационного воздействия может существенно меняться. Под воздействием кавитации в водном растворе, содержащем инертные и активные газы, возможно существование разнообразных химических реакций [14-16].

Кавитационная обработка (в отличие, например, от омагничивания, воздействия различных полей электромагнитного происхождения и т.п.) дает устойчивые повторяющиеся результаты в получении модифицированной в процессе механолиза воды, воспроизводимые независимо от места и времени [1].

Наряду с указанными, в кавитационной полости протекают реакции трансформирования радикалов с участием химически активных газов и рекомбинации радикалов за время $t \cong 10^{-7}-10^{-6}$ с. В результате этих процессов после коллапса кавитационного пузырька в раствор переходят продукты радикального разложения молекул $\mathrm{H}_{2} \mathrm{O}$, обнаруженные с помощью метода спиновых ловушек [17], и рекомбинации радикалов, что приводит к накоплению в воде молекулярного $\mathrm{O}_{2}, \mathrm{H}_{2} \mathrm{O}_{2}, \mathrm{H}_{2}$ и других соединений. Высокая скорость протекания реакций свидетельствует о том, что они происходят непосредственно в зоне схлопывания пузырька.

\section{Физическая модель кавитирующей жидкости}

В гидродинамических процессах с развитой пузырьковой кавитацией, используемых в различных технологиях $[1,15,16]$, наблюдаются сложнейшие пространственные интерференционные картины волн разрежения-сжатия как результат динамики коллапса кавитационных микропузырьков. Одновременно необходимо учитывать быстроменяющиеся поля высоких давлений и температур, а также турбулентные микропотоки, обуславливающие микроперемешивание среды. Анализ ранее полученных результатов свидетельствует о необходимости дальнейших более углубленных исследований с целью расширения сфер применения кавитационной технологии, уточнения исходных данных для расчета и проектирования суперкавитирующих механизмов, уточнения физических и математических моделей процессов кавитационного воздействия на жидкие системы и твердые границы потоков. Здесь сделана попытка реализации новых подходов для решения реологических задач физики кавитации в области использования импульсных воздействий ее пузырьковых форм. 
Свойства потоков при наличии кавитации значительно отличаются от свойств обычных потоков вследствие увеличения объема потока из-за бурного испарения жидкости с образованием кавитационных микропузырьков $[5,18]$. Необходимо учитывать сжимаемость жидкости и усложненные уравнения состояния газа в пузырьках. Кавитация - явление, в котором участвуют несколько агрегатных состояний в развитом турбулентном режиме. В этом случае жидкость возможно рассматривать как своеобразную жидкую среду со структурой, образованной хаотически движущимися и взаимодействующими между собой и потоком молями. Следовательно, возникает необходимость более четкой интерпретации и учета в общем случае изменяющейся вязкости потока. Рассмотрев уравнения Навье-Стокса как запись второго закона Ньютона для моля жидкости, можно сказать, что скорость деформации должна учитывать возможную частоту актов молекулярного взаимодействия, вызванную гидродинамическим фактором относительного послойного смещения. Известно [19], что коэффициент динамической вязкости, учитывающий физико-химическую природу жидкости, должен учитывать размеры и массы молекул, их взаимное расположение, т.е. геометрический объем среды, приходящийся на «возбужденную» молекулу.

\section{Задача сопряжения для пузырька в жидкости}

Формы течений для пузырька в жидкости можно условно разделить на четыре типа: с образованием кумулятивной струйки; «холодное» кипение; газификация пограничного слоя; пульсация парогазового пузырька. В начальной стадии коллапса парогазового пузырька с образованием кумулятивной ультраструйки (по Корнфельду-Суворову, 1944) [20] необходимо учитывать гидро- и термодинамические процессы вне и внутри него. Для парогазовой (внутри пузырька, $i=g$ ) и жидкой (вне пузырька, $i=l$ ) сред можно записать исходные (базовые) уравнения физики (законы сохранения):

$$
\begin{aligned}
& \frac{\partial \rho_{i}}{\partial t}+\left(\vec{V}_{i} \cdot \vec{\nabla}\right) \rho_{i}+\rho_{i} \operatorname{div} \vec{V}_{i}=0 ; \\
& \rho_{i}\left[\frac{\partial \vec{V}_{i}}{\partial t}+\left(\vec{V}_{i} \cdot \vec{\nabla}\right) \vec{V}_{i}\right]=\rho_{i} \vec{F}_{i}+\operatorname{div} \Pi_{i}, \\
& \rho_{i}\left[\frac{\partial U_{i}}{\partial t}+\left(\vec{V}_{i} \cdot \vec{\nabla}\right) U_{i}\right]=\rho_{i} q_{i}^{l}+\Pi_{i}: \dot{S}_{i},
\end{aligned}
$$

где $\Pi_{i}-$ симметричный тензор напряжений; $\rho_{i}-$ массовая плотность; $\vec{V}_{i}-$ скорость; $\vec{F}_{i}-$ плотность газовых сил;

$$
\rho_{i} q_{i}^{l}=\operatorname{div}\left(\lambda_{i} \operatorname{grad} T_{i}^{0}\right)
$$

при передаче тепла лишь теплопроводностью по закону Фурье, $U_{i}-$ внутренняя энергия; $\dot{S}_{i}-$ тензор скоростей деформаций.

Замыкающими для системы (4)-(6) являются уравнения состояния: калорическое:

$$
\rho_{i}=\rho_{i}\left(P_{i}, T_{i}^{0}\right)
$$

для воды по Тэту: 


$$
\begin{aligned}
& \frac{P+B}{P_{0}+B}=\left(\frac{\rho}{\rho_{0}}\right)^{n} ; \\
& \Pi_{i}=\Pi_{i}\left(\dot{S}_{i}\right) ;
\end{aligned}
$$

механическое для ньютоновских жидкостей:

$$
\Pi_{i}=-\left(P_{i}+\frac{2}{3} \mu_{i} \operatorname{div} \vec{V}_{i}\right) I+2 \mu_{i} \dot{S}_{i}
$$

На границе раздела фаз $\Omega_{q l}$ высчитывают условия сопряжения по массе, скорости, импульсу энергии и температуре:

$$
M_{\mathrm{lg}}=\rho_{e} j_{l}=\rho_{l}\left[\frac{-\frac{\partial \Omega_{g l}}{\partial t}+\left(\vec{V}_{l} \cdot \vec{\nabla}\right) \Omega_{g l}}{\left|\vec{\nabla} \Omega_{g l}\right|}\right]=\rho_{g} j_{g}=\rho_{g}\left[\frac{-\frac{\partial \Omega_{g l}}{\partial t}+\left(\vec{V}_{l} \cdot \vec{\nabla}\right) \Omega_{g l}}{\left|\vec{\nabla} \Omega_{g l}\right|}\right],
$$

где $\vec{\tau} \cdot \vec{V}_{l}=\vec{\tau} \cdot \vec{V}_{g}-$ условие прилипания;

$$
\begin{aligned}
& \vec{n} \Pi_{l}+\rho_{l} j_{1} \vec{V}_{l}=\vec{n} \Pi_{g}+\rho_{g} j_{g} \vec{V}_{g}+\vec{n} \sigma_{g l}\left(\frac{1}{M_{g l}}+\frac{1}{N_{g l}}\right) ; \\
& \left(\vec{n} \cdot \Pi_{l}\right) \vec{V}_{l}-\lambda_{l} \frac{\partial T_{l}^{0}}{\partial n}+\rho_{l} j_{1}\left(\frac{V^{2}}{2}+h_{l}\right)=\left(\vec{n} \cdot \Pi_{g}\right) \vec{V}_{g}+q_{g}^{l}+\rho_{g} j_{g}\left(\frac{V_{g}^{2}}{2}+h_{g}\right) ; \\
& T_{l}^{0}=T_{g}^{0}+\Delta T_{g l}^{0} .
\end{aligned}
$$

При задании начальных и граничных условий на твердой стенке $\left(\vec{V}_{l}=0\right), T_{l}^{0}=T_{0}^{0}$ получаем задачу сопряжения для системы сред «жидкость-газ». Частные случаи этих задач со сферической симметрией были получены Си-Дин-Ю (1965), Л.И. Седовым (1970), Р.И. Нигматуллиным (1975-1978), В.М. Ивченко (1971-1977) [21, 22]. Для задач со сферической симметрией решение имеет хорошее совпадение с экспериментальной зависимостью радиуса $R_{0}$ от времени $\tau$ [8]. Однако при рассмотрении данной задачи не все физические переменные были учтены (например, такие, как возрастание давления паров при $R<<R_{\max }$, отклонение от сферичности вблизи стенок, влияние вязкости и др.). В этой связи имеем лишь качественно правильную картину.

Используя подход к рассмотрению турбулентных потоков, описанный в [23, 24], попытаемся уточнить влияние вязкости в кавитационных течениях. Влияние вязкости сводится к демпфированию и связано с диссипацией механической энергии в процессе роста и схлопывания пузырьков. Такие расчеты в несжимаемой жидкости с учетом поверхностного натяжения были выполнены H. Poritsky [19] и обнаружили существенное влияние вязкости при значениях, заметно превышающих вязкость воды в обычных условиях. По методу [19] движение стенки пузырька описывают уравнением

$$
\frac{P_{i}-p_{\infty}}{\rho}=R \ddot{R}+\frac{3}{2} \dot{R}^{2}+4 \frac{\mu}{\rho} \frac{1}{2} \dot{R}
$$

Из формулы (17) можно получить уравнение энергии

$$
-823-
$$




$$
\frac{\left(P_{i}-p_{\infty}\right)\left(R_{0}^{3}-R\right)}{3}+\frac{\rho}{2} R^{3} \dot{R}^{2}+4 \mu \int_{0}^{t} R \dot{R}^{2} d t=0,
$$

где последний член учитывает диссипацию энергии вследствие вязкости (на один стерадиан). Время схлопывания, определенное по (17), для пузырька без поверхностного натяжения становится бесконечно большим, если параметр

$$
\mu^{\prime}=\frac{4 \mu}{R_{0} \sqrt{\rho\left(p_{\infty}-P_{i}\right)}}
$$

превышает критическое значение, равное 0,46.

В работе Р.Д. Айвени [25] вязкость и поверхностное натяжение учитывали в сжимаемой жидкости. Был использован метод H. Poritsky, который F.R. Gilmore [26] применил в условиях сжимаемости, основываясь на гипотезе J.G. Kirkwood - H.A. Bethe [27]. Вязкость и поверхностное натяжение учитывали в граничном условии для давления в жидкости с помощью уравнения

$$
P=P_{i}-\frac{2 \sigma}{R}-4 \mu \frac{1}{2} \dot{R}
$$

Согласно [25], вязкость и поверхностное натяжение не влияют на общий характер поведения каверны. Кажущиеся противоречия его результатов детально проанализированы в [2]. Результаты последующих работ, обобщенные в [5, 22, 28, 29 и др.], показывают, что вязкость, входящая в уравнение Навье-Стокса в виде коэффициента динамической вязкости, может быть учтена более или менее удачно. Однако физическая интерпретация этого коэффициента недостаточна [30].

Условимся, как это сделано в [30] на основании работ [31, 32], под частицами жидкости понимать молекулы, надмолекулярные образования, ассоциаты и более крупные образования как турбулентные вихри. Тогда и кавитационные микропузырьки и микровихри, образующиеся в результате их коллапса, можно ассоциировать с частицами жидкости. Исходя из этого, можно сформулировать следующую феноменологическую модель кавитирующей жидкости (в отсутствие твердых границ потока): кавитационный микропузырек в своей динамике движется в пространстве, структурированном микротурбулентными вихрями, образованными интерференцией волн разрежения-сжатия, возникающих в результате пульсации кавитационных микропузырьков.

Для изотропной среды (стохастически и статистически принимая поля кавитационных микропузырьков изотропной средой), физических констант, выражающих ее свойства, существует связь между тензором напряжений и скоростей деформации в виде соотношения $[33]$

$$
\Pi=a \dot{S}+b I
$$

где $a$ и $b$ - скаляры. Скаляр $a$ представляет собой физическую константу, которая из условия совпадения (21) со своим частным случаем, законом жидкостного трения Ньютона

$$
\tau=\mu \frac{d \vec{V}}{d n}
$$


полагается равной $2 \mu$. Скаляр $b$ может быть линейно связан с П и $\dot{S}$ через их линейные инварианты.

С помощью действий альтернирования и симметрирования уравнение движения в напряжениях возможно разбить на два, как это сделано в [30]:

$$
\begin{aligned}
& \nabla_{k}\left(\Pi_{k i}\right)=\nabla_{k}\left[\frac{1}{2} \rho\left(V_{d k} \dot{S}_{i j} \delta_{r j}+V_{d i} \dot{S}_{k j} \delta_{r j}\right)\right]-\nabla_{k}\left(\Pi I_{k i}\right), \\
& \nabla_{k}\left(\Pi_{k i}\right)_{*}=\nabla_{k}\left[\frac{1}{2} \rho\left(V_{d k} \dot{S}_{i j} \delta_{r j}+V_{d i} \dot{S}_{k j} \delta_{r j}\right)\right]+\rho I_{i j k} \omega_{k} \delta_{r j},
\end{aligned}
$$

где $\mathrm{V}_{\mathrm{d}}$ - скорость деформации движения; $\delta_{r j}-$ элементарный отрезок; $\omega$ - угловая скорость.

Первое из этих уравнений характеризует движение сплошной среды в случае «симметричной» гидродинамики, а уравнение (24) - «несимметричной», когда в жидкости присутствуют непрерывно распределенные пары сил. Из уравнения (23) следует новое реологическое уравнение

$$
\Pi_{k i}=\frac{1}{2}\left(\rho V_{d k} \delta_{r j} \dot{S}_{i j}+\rho V_{d i} \delta_{r j} \dot{S}_{k j}\right)-\Pi I_{k i}
$$

или

$$
\Pi_{k i}=\rho V_{d k} V_{d i}-\Pi I_{k i}=\rho \dot{S}_{k j} \dot{S}_{i j}\left(\delta_{r j}\right)^{2}-\Pi_{k i} .
$$

Применительно к (25) вводится обозначение

$$
M_{k j}=\frac{1}{2} \rho V_{d k} \delta r_{j}=\frac{1}{2} \rho \dot{S}_{k j}\left(\delta r_{j}\right)^{2}
$$

Размерность тензора $M_{k j}$ совпадает с размерностью коэффициента динамической вязкости $\mu$ в (22). По физическому смыслу $M_{k j}$ описывает внутренний момент импульса элемента жидкости, появляющийся вследствие его деформации при движении. Тогда

$$
\Pi_{k i}=\left(M_{k j} \dot{S}_{i j}+M_{k j} \dot{S}_{k j}\right)-\Pi I_{k i}=2 M_{k j} \dot{S}_{i j}-\Pi I_{k i} .
$$

В случае анизотропии переноса импульса и вещества с учетом (28) уравнение движения в напряжениях принимает вид

$$
\rho_{i} \frac{d \vec{V}_{i}}{d t}=\rho_{i} \vec{F}_{i}+2 \vec{V}_{k}\left(M_{k j} \dot{S}_{i j}-\Pi_{i} I_{k i}\right)
$$

При задании тензора напряжений одним из выражений (25), (26) или (28) в уравнении Навье-Стокса (29) дополнительная вязкость как фактор регуляризации проявляется в любом сдвиговом течении (например, введение дополнительной вязкости необходимо для удержания детерминированности процесса, особенно в области больших скоростей [34]).

\section{Заключение}

Таким образом, задача сопряжения для пузырька в жидкости может быть замкнута новым реологическим уравнением или задана уравнением (29) с учетом тензора вязкости. Решение 
для случая динамики сферического пузырька с учетом новой постановки задачи приводит к более точному совпадению с результатами эксперимента.

В итоге наряду с микротурбулентным перемешиванием и активацией поверхности водных полуфабрикатов процесс механолиза воды при ее гидромеханической обработке позволяет создавать и использовать кавитационную технологию для интенсификации различных технологических процессов и служит основой для разработки новых приложений [35-39 и др.].

\section{Благодарность}

Исследование выполнено при финансовой поддержке РФФИ и Правительства Красноярского края в рамках научных проектов № 18-48-242001 «Теплофизические и гидродинамические особенности кинетики смесеобразования при иммобилизации радиоактивных отходов в цементную матрицу с использованием эффектов кавитации», № 18-41-242004 «Теоретические основы кондиционирования вод питьевого назначения на базе эффектов гидродинамической кавитации».

\section{Список литературы}

[1] Кулагин В.А. Методы и средства технологической обработки многокомпонентных сред с использованием эффектов кавитации: автореф. дис. ... д-ра техн. наук: 01.04.14, 01.02.05. Красноярск: КГТУ, 2004. 47 с. [Kulagin V.A. Methods and means of technological processing of multicomponent media using cavitation effects: author. dis. ... Dr. tech. Sciences: 01.04.14, 01.02.05. Krasnoyarsk, KSTU, 2004. 47 p. (in Russian)]

[2] Кнепп Р., Дейли Дж., Хеммит Ф. Кавитация. М.: Мир, 1974. 688 с. [Knepp R., Daily J., Hemmit F. Cavitation. M.: Mir, 1974. 688 p. (in Russian)]

[3] Тирувенгадам А. Обобщенная теория кавитационных разрушений. Tpyды ASME, cep. D. Техническая механика, 1969, 3, 48-62 [Tiruvengadam A. The generalized theory of cavitation destruction. Proceedings of ASME, ser. D. Technical Mechanics, 1969, 3, 48-62 (in Russian)]

[4] Hobbs J.M. Experience with a 20kc Cavitation Erosion Test. Erosion by Cavitation or Impingement. Atlantic City. ASTM, STR, 1967, 408, 159-185.

[5] Ивченко В.М., Кулагин В.А., Немчин А.Ф. Кавитацчионная технология; ред. акад. Г.В. Логвинович. Красноярск: Изд-во КГУ, 1990. 200 с. [Ivchenko V.M., Kulagin V.A., Nemchin A.F. Cavitation technology; ed. Acad. G.V. Logvinovich. Krasnoyarsk: KSU Publishing House, 1990. 200 p. (in Russian)]

[6] Демиденко Н.Д., Кулагин В.А., Шокин Ю.И., Ли Ф.-Ч. Тепломассообмен и суперкавитация. Новосибирск: Наука, 2015. 436 с. [Demidenko N.D., Kulagin V.A., Shokin Yu.I., Lee F.-Ch. Heat and mass transfer and supercavitation. Novosibirsk: Nauka, 2015. 436 p. (in Russian)]

[7] Демиденко Н.Д., Кулагин В.А., Шокин Ю.И. Моделирование и вычислительные технологии распределенных систем; ред. чл.-корр. РАН А.М. Федотов. Новосибирск: Наука, 2012. 424 c. [Demidenko N.D., Kulagin V.A., Shokin Yu.I. Modeling and computing technology of distributed systems; ed. Corr. RAS A.M. Fedotov. Novosibirsk: Nauka, 2012. 442 p. (in Russian)]

[8] Кулагин В.А., Вильченко А.П., Кулагина Т.А. Моделирование двухфазных суперкавитационных потоков; ред. В.И. Быков. Красноярск: ИПЦ КГТУ, 2001. 187 с. [Kulagin V.A., 
Vilchenko A.P., Kulagina T.A. Simulation of two-phase supercavitation flows; ed. V.I. Bikov. Krasnoyarsk: CPI KSTU, 2001. 187 p. (in Russian)]

[9] Маргулис М.А. Звукохимические реакиии и сонолюминесиенция. М.: Химия, 1986. 288 с. [Margulis M.A. Sound chemical reactions and sonoluminescence. M.: Chemistry, 1986. 288 p. (in Russian)]

[10] Мёрч К.Д. Динамика кавитационных пузырьков и кавитационных жидкостей. Эрозия; ред. К. Прис. М.: Мир, 1982, 331-382 [Merch K.D. The dynamics of cavitation bubbles and cavitation fluids. Erosion; ed. K. Pris. M. Mir, 1982, 331-382 (in Russian)]

[11] Балабышко А.М., Зимин. А.И., Ружицкий В.П. Гидромеханическое диспергирование. М.: Наука, 1998. 331 с. [Balabyshko A.M., Zimin. A.I., Ruzhitsky V.P. Hydromechanical dispersion. M.: Nauka, 1998. 331 p. (in Russian)]

[12] Маргулис М.А., Мальцев А.Н. Об оценке энергетического выхода химических реакций, инициированных ультразвуковыми волнами. ЖФХ, 1968, 42, 1441-1447 [Margulis M.A., Maltsev A.N. On the evaluation of the energy yield of chemical reactions initiated by ultrasonic waves. Physical Chemistry Journal, 1968, 42, 1441-1447 (in Russian)]

[13] Зацепина Г.Н. Физические свойства и структура воды. М.: Изд-во МГУ, 1987. 171 с. [Zatsepina G.N. Physical properties and structure of water. M.: Publishing House of Moscow State University, 1987. 171 p. (in Russian)]

[14] Механика многокомпонентных сред в технологических процессах. Отделение механики и проиессов управления; отв. ред. акад. В.В. Струминский; АН СССР. М.: Наука, 1978. 148 c. [Mechanics of multicomponent media in technological processes. Department of Mechanics and Control Processes; Repl. ed. Acad. V.V. Struminsky; USSR Academy of Sciences. M.: Nauka, 1978. 148 p. (in Russian)]

[15] Розен А.М. Масштабный переход в химической технологии. Разработка промышленных аппаратов методомгидродинамического моделирования. М., 1980. 200 c. [Rosen A.M. Largescale transition in chemical technology. Development of industrial apparatuses by hydrodynamic modeling. M., 1980. 200 p. (in Russian)]

[16] Кафаров В.В., Винаров А.Ю., Гордеев Л.С. Моделирование и системный анализ биохимических производств. М., 1985. 280 с. [Kafarov V.V., Vinarov A.Yu., Gordeev L.S. Modeling and system analysis of biochemical industries. M., 1985. 280 p. (in Russian)]

[17] Wang T. Effects of evaporatyion and diffusion or an oscillating bubble. The Physics of Fluids, 1974, 17(6), 1121-1126.

[18] Кулагин В.А., Москвичев В.В., Махутов Н.А., Маркович Д.М., Шокин Ю.И. Проблемы физического и математического моделирования в области гидродинамики больших скоростей на экспериментальной базе Красноярской ГЭС. Вестник Российской академии наук, 2016, 86(11), 978-990. DOI: 10.7868/S0869587316110062 [Kulagin V.A., Moskvichev V.V., Makhutov N.A., Markovich D.M., Shokin Yu.I. Physical and Mathematical Modeling in the Field of High-Velocity Hydrodynamics in the Experimental Base of the Krasnoyarsk Hydroelectric Plant. Herald of the Russian Academy of Sciences, 2016, 86(6), 454-465; DOI: 10.1134/ S1019331616060034 (in Russian)]

[19] Poritsky H., Chapmen R.B. Collaps or Growth of a Spherical Bubble or Cavity in a Viscous Fluid. Proc. First U. S. Natl. Congr. Appl. Mech. (ASME), 1952, 813-821.

$$
-827-
$$


[20] Корнфельд М. Упругость и прочность жидкостей. М.-Л.: ГИТТЛ, 1951. 107 с. [Kornfeld M. Elasticity and strength of liquids. M.-L.: GITTL, 1951. 107 p. (in Russian)]

[21] Нигматуллин Р.И. Основы механики гетерогенных сред. М.: Наука, 1972. 336 с. [Nigmatullin R.I. Fundamentals of the mechanics of heterogeneous media. M.: Nauka, 1972. 336 p. (in Russian)]

[22] Ивченко В.М. Гидродинамика суперкавитирующих механизмов. Иркутск: Изд-во Иркут. ун-та, 1985. 232 с. [Ivchenko V.M. Hydrodynamics of super-cavitating mechanisms. Irkutsk: Publishing house Irkut. University, 1985. 232 p. (in Russian)]

[23] Thiruvengadam A. Scaling Law for Cavitation Erosionc. Неустановивииеся течения воды с большими скоростями: Труды JUTAM. М.: Наука, 1973, 405-427 [Thiruvengadam A. Scaling Law for Cavitation Erosionc. Unsteady flows of water at high speeds: Proceedings of JUTAM. M.: Nauka, 1973, 405-427]

[24] Plesset M.S., Chapman R.B. Collapse of an Initially Spherical Vapour in the Neighbourhood of a Solid Boundary. Journal of Fluid Mechanics, 1971, 47(2), 125-141.

[25] Айвени Р.Д., Хэммит Ф.Г. Численный анализ явления схлопывания кавитационного пузырька в вязкой жидкости. Tp. ASME. Cер. D: Tеоретические методы инженерных расчетов, 1965, 4, 140 [Aveni R.D., Hammit F.G. Numerical analysis of the phenomenon of collapse of a cavitation bubble in a viscous fluid. Tr. ASME. Ser. D: Theoretical methods of engineering calculations, 1965, 4. 140 (in Russian)]

[26] Gilmore F.R. The Growth and Collaps of a Spherical Bubble in a Viscous Compressible Liquid. Rept 26-4, Calif. Inst. Of Tech. Hydrodyn, 1952.

[27] Kirkwood J.G., Bethe H.A. The Pressure Wave Produced by an Underwater Explosion. OSRD Rept 588, 1942.

[28] Биркгоф Г., Сарантонелло Э. Струи, следы и каверны. М.: Мир, 1964. 466 c. [Birkhoff G., Sarantonello E. Streams, traces and caverns. M.: Mir, 1964. 466 p. (in Russian)]

[29] Седов Л.И. Механика сплошной среды. Т. 1, 2. М.: Наука, 1973. 536 с., 584 с. [Sedov L.I. Continuum mechanics. Vol. 1 and 2. M.: Nauka, 1973. 536 p., 584 p. (in Russian)]

[30] Никулин В.А. Основные уравнения движения реальных жидкостей. Гидродинамика течений с тепломассообменом. Устинов: УМИ, 1986. С. 4-15 [Nikulin V.A. The basic equations of motion of real liquids. Hydrodynamics of flows with heat and mass transfer. Ustinov: UMI, 1986. P. 4-15 (in Russian)]

[31] Репин Н.Н., Телевин Л.А. Возникновение турбулентности. Уфа: Башкирское кн. изд., 1977. 96 c. [Repin N.N., Televin L.A. The emergence of turbulence. Ufa: Bashkir book ed., 1977. 96 p. (in Russian)]

[32] Скворцов Г.Е., Тимохов Л.А. К теории турбулентности. Вестник ЛГУ, 1980. 2(13), 106-110 [Skvortsov G.E., Timokhov L.A. To the theory of turbulence. Bulletin of Leningrad State University, 1980, 2(13), 106-110 (in Russian)]

[33] Лойцянский Л.Г. Механика жидкости и газа. М.: Наука, 1978. 736 c. [Loytsyansky L.G. Mechanics of fluid and gas. M.: Nauka, 1978. 736 p. (in Russian)]

[34] Ладыженская О.А. Математические вопросы динамики вязкой несжимаемой жидкости. М.: Наука, 1970. 288 с. [Ladyzhenskaya O.A. Mathematical problems of the dynamics of a viscous incompressible fluid. M.: Nauka, 1970. 288 p. (in Russian)] 
[35] Kulagina T., Kulagin V., Nikiforova E., Prikhodov D., Shimanskiy A. and Li F. Inclusion of liquid radioactive waste into a cement compound with an additive of multilayer carbon nanotubes. IOP Conf. Series: Earth and Environmental Science 227 (2019) 052030; DOI: 10.1088/17551315/227/5/052030.

[36] Zhi-Ying Zheng, Qian Li, Lu Wang, Li-Ming Yao, Wei-Hua Cai, Vladimir A. Kulagin, Hui Li, Feng-Chen Li Numerical study on the effect of steam extraction on hydrodynamic characteristics of rotational supercavitating evaporator for desalination [J]. Desalination 455 (2019) 1-18; DOI. org/10.1016/j.desal.2018.12.012 (IF 6,603, Q1).

[37] Махутов Н.А., Москвичев В.В., Кулагин В.А. Современные технологии топливноэнергетического комплекса и возможности развития железнодорожного транспорта Сибири. Современныетехнологии. Системныйанализ. Моделирование, 2019, 61(1), 64-73; DOI: 10.26731/18139108.2019.1(61). 64-73 [Makhutov N.A., Moskvichev V.V., Kulagin V.A. Modern technologies of the fuel and energy complex and the possibility of the Siberia railway transport development. Modern Technologies. System Analysis. Modeling, 2019, 61(1), 64-73. DOI: 10.26731/1813-9108.2019.1(61). 64-73 (in Russian)]

[38] Dubrovskaya O.G., Kulagin V.A. Non-reagent cleaning of industrial wastewater, containing heavy metals based on technology of hydrothermodynamic cavitation, J. Sib. Fed. Univ. Eng. technol., 2019, 12(4), 460-467. DOI: 10.17516/1999-494X-0153.

[39] Kulagin V.A. et. al. The Technology of Obtaining Modified Sorbents Based on Silicate Production Waste. IOP Conf. Ser.: Earth Environ. Sci. 288 (2019) 012013; DOI: 10.1088/1755$1315 / 288 / 1 / 012013$ 